\title{
Distribution of cerebral blood flow in the caudate nucleus, lentiform nucleus and thalamus in patients with carotid artery stenosis
}

\author{
Nolan S. Hartkamp • Reinoud P. H. Bokkers • \\ H. Bart van der Worp • M. J. P. van Osch • \\ L. Jaap Kappelle • Jeroen Hendrikse
}

Received: 24 May 2010 /Revised: 1 August 2010 / Accepted: 17 August 2010 /Published online: 18 September 2010

(C) The Author(s) 2010. This article is published with open access at Springerlink.com

\begin{abstract}
Objective To investigate the influence of internal carotid artery (ICA) stenosis on the distribution of blood flow to the caudate nucleus, lentiform nucleus, and thalamus.

Methods We studied 18 healthy control subjects, 20 patients with a unilateral asymptomatic ICA stenosis, and 15 patients with a recently symptomatic unilateral ICA stenosis. The contribution of the ICAs and the basilar artery to the perfusion of the deep brain structures was assessed by perfusion territory selective arterial spin labeling (ASL) MRI. Differences were tested with a two-tailed Fishers' exact test.

Results The caudate nucleus was predominantly supplied with blood by the ipsilateral ICA in all groups. In 4 of the $15(27 \%)$ the symptomatic patients, the caudate nucleus partially received blood from the contralateral ICA, compared to none of the 18 healthy control subjects ( $p=$ 0.03 ). The lentiform nucleus and the thalamus were predominantly supplied with blood by the ipsilateral ICA and basilar artery respectively in all groups.
\end{abstract}

\footnotetext{
N. S. Hartkamp $(\bowtie) \cdot$ R. P. H. Bokkers · J. Hendrikse

Department of Radiology, University Medical Center Utrecht,

PO Box 85500, 3508 GA Utrecht, The Netherlands

e-mail: n.s.hartkamp@umcutrecht.nl
}

H. B. van der Worp · L. J. Kappelle

Department of Neurology, Rudolf Magnus Institute

of Neuroscience, University Medical Center Utrecht,

Utrecht, The Netherlands

M. J. P. van Osch

Department of Radiology, C.J. Gorter Institute of High Field MRI, Leiden University Medical Center,

Leiden, The Netherlands
Conclusion In patients with a symptomatic ICA stenosis, the caudate nucleus may be supplied with blood by the contralateral ICA more often than in healthy controls.

Keywords Magnetic resonance imaging . Cerebral hemodynamics $\cdot$ Perfusion $\cdot$ Basal ganglia . Carotid artery stenosis

\section{Introduction}

There is wide variation in the perfusion territories of the brain-feeding arteries, which is most pronounced in those supplying the deep brain structures [1-3]. The caudate nucleus, lentiform nucleus, and thalamus are supplied with blood from groups of different perforating arteries that branch either from the anterior, middle and posterior cerebral artery or from the posterior communicating artery $[2,4-7]$. In addition to the normal variability, there may be a shift in the perfusion territories of the brain-feeding arteries in patients with steno-occlusive disease of the ICA, due to the recruitment of collateral blood pathways $[8,9]$. Perfusion territory selective arterial spin labeling magnetic resonance imaging (ASL-MRI) has recently been introduced to visualize the perfusion territories of the individual brain-feeding arteries $[10,11]$. ASL-MRI is a non-invasive technique for imaging whole-brain cerebral perfusion that uses radiofrequency pulses to magnetically label blood and does not require gadolinium-based contrast agents.

The aim of our study was to investigate the influence of ICA stenosis on the distribution of cerebral blood flow to the caudate nucleus, lentiform nucleus and thalamus with perfusion territory selective ASL-MRI in patients with 
either symptomatic or asymptomatic ICA stenosis, and to compare this distribution with that of healthy control subjects.

\section{Materials and methods}

The study was approved by the institutional ethical review board and written informed consent was obtained from all participants.

\section{Subjects}

Fifty-three subjects were included prospectively in the study. Thirty-five were functionally independent patients with a unilateral ICA stenosis of $\geq 50 \%$ and 18 were healthy control subjects. Of the patients, 20 had an asymptomatic ICA stenosis (12 males and 8 females; mean age $\pm \mathrm{SD}, 69 \pm 7$ years) and 15 a symptomatic stenosis ( 9 male, 6 female; $71 \pm 7$ years). The symptomatic patients had experienced a transient ischemic attack (TIA), non-disabling ischemic stroke, or amaurosis fugax on the side of the stenosis in the 3 months before inclusion and were seen at our hospital for diagnosis and treatment. The patients with an asymptomatic ICA stenosis were recruited from a hospital-based vascular screening study involving subjects with risk factors for atherosclerosis or clinically manifest atherosclerotic vessel disease [12]. Stenoses of the ICAs were measured with color Doppler-assisted Duplex ultrasonography and peak systolic velocity thresholds based on literature were used for the diagnosis of less than $50 \%, 50 \%$ to $70 \%$ or $70 \%$ to $99 \%$ stenoses [13]. The control group consisted of 18 healthy age and sex-matched volunteers (11 male, 7 female; $67 \pm 7$ years). All control subjects were recruited through local media advertisements and did not have a history of neurological disease or vascular pathology on MRI or MR angiography (MRA) of the brain. Subjects with diabetes mellitus or severe renal or liver dysfunction were excluded.

\section{Magnetic resonance imaging}

Each subject underwent an MRI investigation on a clinical 3 Tesla MRI system (Achieva, Philips Medical Systems, The Netherlands) equipped with an eight-element phasedarray head coil and locally developed software for ASLMR imaging. To depict the perfusion territories of the ICAs and the basilar artery, territory specific perfusion images were acquired with a pseudo-continuous selective ASL perfusion sequence, according to a previously published protocol $[14,15]$. To position the imaging section, a $\mathrm{T}_{1}$-weighted spin-echo sequence was first obtained in the sagittal plane. Seventeen $7 \mathrm{~mm}$ slices with no slice gap were subsequently aligned parallel to the orbito-meatal angle, as shown in Fig. 1a. Labeling was performed by employing a train of $18^{\circ}, 0.5 \mathrm{~ms}$, Hanning-shaped radiofrequency pulses at an interpulse pause of $0.5 \mathrm{~ms}$, for a duration of $1,650 \mathrm{~ms}$, in combination with a balanced gradient scheme $[16,17]$. The control situation, without labeling of arterial blood, was achieved by adding $180^{\circ}$ to the phase of all even RF pulses. Perfusion territory selective ASL-MRI was accomplished through manipulating the spatial labeling efficiency by applying additional gradients between the labeling pulses in sets of 5 dynamics as demonstrated in Fig. 1b. Imaging was performed with single-shot echo planar imaging in combination with parallel imaging (SENSE factor 2.5) and background suppression. The background suppression consisted of a saturation pulse immediately before labeling and inversion pulses at 1,680 and 2,830 $\mathrm{ms}$ after the saturation pulse, 1,525 $\mathrm{ms}$ after the labeling stopped [18]. The following parameters were used: repetition time (TR), 4,000 ms; echo time (TE), $14 \mathrm{~ms}$; field of view (FOV), $240 \times 140 \mathrm{~mm}$; matrix size, $89 \times 79 ; 75$ dynamics; scan time, 51/2 min.

A three-dimensional time-of-flight MR angiography was made with subsequent maximum intensity projection reconstruction using the following parameters: TR/TE, 30/6.9 ms; flip angle $20^{\circ} ; 2$ averages; FOV, $100 \times 100 \mathrm{~mm}$; matrix, 256× 256; 50 slices; slice thickness, $1.2 \mathrm{~mm}$ with $0.6 \mathrm{~mm}$ overlap; scan time, $3 \mathrm{~min}$. Collateral blood flow direction was determined according to a previously published imaging protocol with 2 consecutive 2-dimensional phase-contrast MRI measurements, out of which one was phase-encoded in

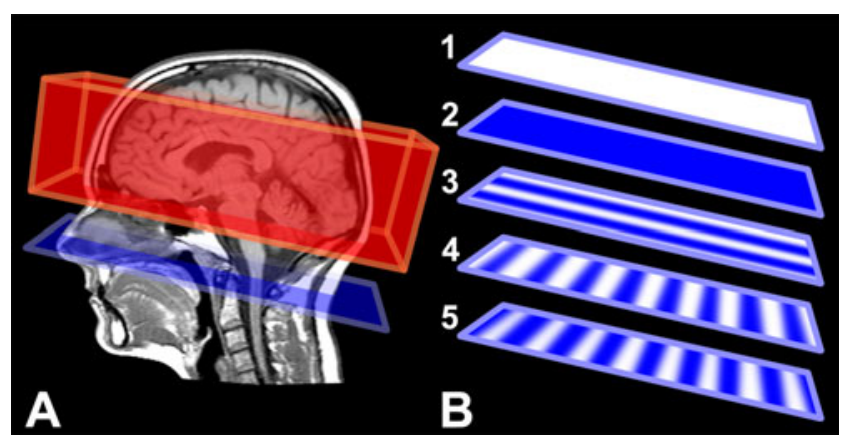

Fig. 1 Sagittal T1 weighted image a illustrating the imaging volume aligned parallel to the orbito-meatal angle and the labeling plane of the regional-perfusion selective ASL imaging. The labeling efficiency (b, white represents no label, blue represents full label) was spatially manipulated within the labeling plane in sets of five dynamics, in which: (1) no labeling applied (control), (2) non-selective labeling applied (globally perfusion weighted), (3) labeling varied in right-left (RL) direction (distance of $50 \mathrm{~mm}$ between full label and control situation), (4) labeling varied in anterior-posterior (AP1) direction (distance of $18 \mathrm{~mm}$ between full label and control situation), and (5) labeling varied in anterior-posterior direction (AP2, similar to AP1, but shifted $9 \mathrm{~mm}$ in posterior direction compared to the previous dynamic) 
the anterior-posterior direction and one in the right-left direction (TR/TE, 9.4/5.9 ms; flip angle, 7.5 ; FOV, 250× 187.5; single slice, 8 averages; slice thickness, $13 \mathrm{~mm}$; velocity sensitivity, $40 \mathrm{~cm} / \mathrm{s}$; scan time, $20 \mathrm{~s}$ ) [19].

Perfusion territory and collateral blood flow assessment

The decision whether the caudate nucleus, lentiform nucleus and thalamus were supplied by the ipsilateral ICA, the contralateral ICA or the basilar artery was made by one author $(\mathrm{NH})$, blinded to the MR angiography images, on the perfusion territory selective ASL-MR images. For this purpose the perfusion territories of the left ICA, right ICA and basilar artery were first identified using k-means clustering with Matlab (The MathWorks, Natick, Mass, version 7.5) [20]. Perfusion contributions were then scored by evaluating the head portion of the caudate nucleus, the entire lentiform nucleus, and entire thalamus. The ventricular system, anterior limb and posterior limb of the internal capsule, and external capsule were used to delineate the deep brain structures.

The morphology of the circle of Willis and the direction of blood flow through the primary collaterals were determined separately, blinded to the perfusion territory selective ASL-MR images, with the time-of-flight and phase-contrast MR angiography images. Anterior collateral flow was defined as blood flow across the anterior communicating artery with retrograde flow in the precommunicating part of the anterior cerebral artery (A1 segment) ipsilateral to the stenosis. Posterior-to-anterior blood flow in the posterior communicating artery was considered to represent posterior collateral flow. Each circle of Willis was assessed for a missing or hypoplastic A1 segment and for the presence of a fetal-type posterior communicating artery, of which the diameter is larger than the precommunicating segment of the ipsilateral posterior cerebral artery.

SPSS (SPSS Inc., Chicago, Illinois, USA version 15) was used for statistical analyses. The frequencies of supply by a specific artery were compared between the symptomatic patients, asymptomatic patients and healthy control subjects with a two-tailed Fisher's exact test. A $p$-value $<0.05$ was considered to indicate statistical significance. Values are expressed as mean \pm standard deviation (SD).

\section{Results}

The demographic and clinical characteristics of the participants are outlined in Table 1. Figure 2 shows the perfusion territories of the caudate nucleus, lentiform nucleus and thalamus, and the corresponding blood flow through the circle of Willis of a patient with a symptomatic right-sided ICA stenosis. Table 2 summarizes the prevalences of various perfusion patterns of the caudate nucleus, lentiform nucleus and thalamus in the healthy control subjects, symptomatic patients and asymptomatic patients. In some patients the deep brain structures received blood from more than one brain-feeding artery.

The caudate nucleus was predominantly supplied by the ipsilateral ICA in all healthy control subjects and symptomatic patients, and in 19 (95\%) of the 20 asymptomatic patients. The caudate nucleus was supplied by the contralateral ICA in one patient (5\%) with an asymptomatic ICA stenosis. In patients with a symptomatic ICA stenosis the caudate nucleus received additional perfusion from the contralateral ICA in $4(27 \%)$ of the 15 patients, whereas this was not seen in healthy controls $(p=0.03)$. There was no difference in the supply of the caudate nucleus between patients with an asymptomatic and patients with a symptomatic ICA stenosis, and between the asymptomatic patients and the healthy control subjects.

The lentiform nucleus was supplied by the ipsilateral ICA in all groups. In one of the 18 healthy control subjects and in one of the 15 patients with a symptomatic ICA stenosis, the lentiform nucleus received additional blood flow contribution from the basilar artery. The thalamus was predominantly supplied by the basilar artery. The ipsilateral and contralateral ICA supplied the thalamus with blood in respectively 17 and one of the 18 healthy control subjects, 12 and 3 of the 15 symptomatic patients, and 15 and 2 of the 20 asymptomatic patients.

In 4 of the $5(80 \%)$ subjects in whom the caudate nucleus received blood from the contralateral ICA, there was anterior collateral flow, compared to 1 of 48 subjects $(2 \%)$ without a contributing contralateral ICA $(p<0.01)$. The collateral flow distribution in the circle of Willis did not affect the perfusion territories of the lentiform nucleus and thalamus between subjects with or without anterior or posterior collateral flow. Regarding the anterior collateral circulation, there were no subjects with a missing or hypoplastic A1 segment. Two asymptomatic patients and one symptomatic patient showed a unilateral fetal-type posterior communicating artery. In these three patients, the thalamus was supplied by the ipsilateral ICA. Figure 3 is an example of the perfusion territories and anatomical morphology of the circle of Willis in a symptomatic patient with a right-sided ICA stenosis and a right-sided unilateral fetal-type posterior communicating artery.

\section{Discussion}

The current study shows that in patients with a symptomatic stenosis of the ICA, the caudate nucleus is more often supplied with blood from the contralateral ICA via collaterals than in healthy control subjects. Secondly, the contribution of 
Table 1 Demographic and clinical characteristics of the study groups

\begin{tabular}{llll}
\hline & Healthy controls & \multicolumn{2}{l}{ Patients with internal carotid stenosis } \\
\cline { 3 - 4 } & & Symptomatic & Asymptomatic \\
\hline Number & 18 & 15 & 20 \\
Male (\%) & $11(61.1 \%)$ & $9(60.0 \%)$ & $12(60.0 \%)$ \\
Age (mean years \pm SD) & $67.4 \pm 6.5$ & $70.9 \pm 7.2$ & $68.7 \pm 6.6$ \\
Degree of ICA stenosis (n) & & & 0 \\
$0-49 \%$ & 18 & 0 & 10 \\
$50-69 \%$ & 0 & 3 & 10 \\
$70-99 \%$ & 0 & 12 & 0 \\
Occluded & 0 & 0 & - \\
Presenting events (n) & - & & - \\
Transient ischemic attack & - & 9 & - \\
Ischemic stroke & - & 3 & \\
Retinal ischemia & & & \\
\hline
\end{tabular}

the contralateral ICA to the caudate nucleus in these patients is accompanied with anterior collateral flow, confirming that blood is being diverted from the contralateral ICA via the circle of Willis. We did not find differences between the three groups with respect to the feeding arteries of the lentiform nucleus and the thalamus.

Knowledge of the perfusion territories of the brainfeeding arteries in the deep brain structures is largely based on post-mortem studies in which dyed resins were injected into the brain-feeding arteries $[2,5]$. With modern imaging modalities such as CT and MR angiography the cerebral vasculature and corresponding perfusion territories can be visualized in vivo. These techniques however can only visualize the vascular tree. Selective ASL-MRI has shown to reliably image the cortical supply of individual major brain-feeding arteries [21]. With the introduction of perfusion territory selective ASL-MRI, the perfusion territories of the brain-feeding arteries can now be visualized at brain tissue level.

Our findings dispute the textbook concept of a fixed pattern of the perfusion territories from the brain-feeding arteries. In a symptomatic patient with steno-occlusive carotid artery disease, information about the individual perfusion territories might be used complementary to the type and anatomical location of ischemic infarction for determining the involved artery. With respect to the deep brain structures, perfusion territory selective ASL-MRI could show if an ischemic area receives blood from the ipsilateral or contralateral ICA (anterior circulation), or from the basilar artery (posterior circulation). Knowledge on the artery perfusing the ischemic area may have consequences for treatment. For instance if a severe stenosis is present that may be amenable by endarterectomy or stenting.

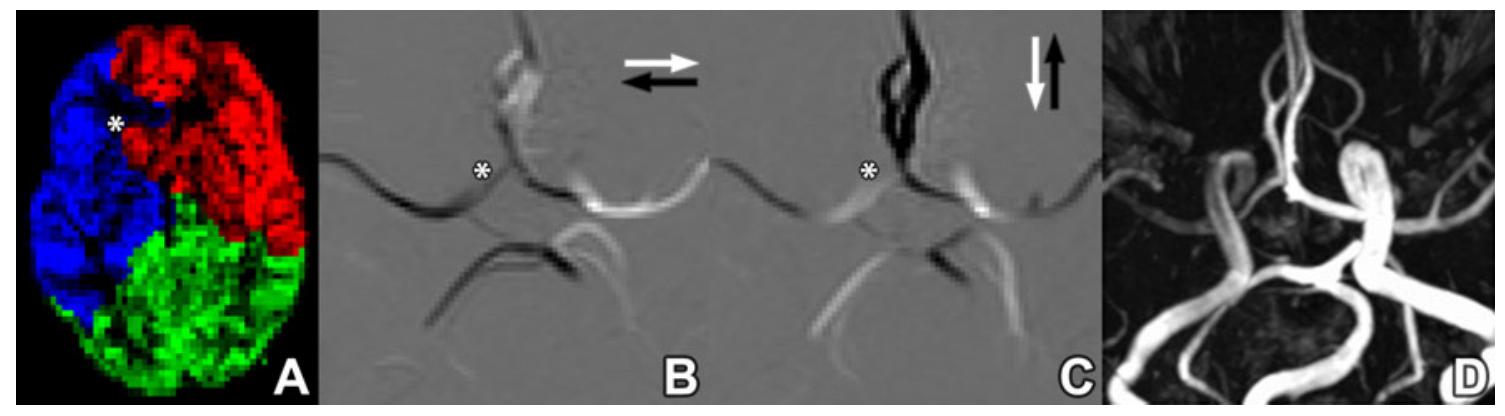

Fig. 2 Perfusion territory image a of a patient with a right-sided symptomatic ICA stenosis. Colors represent the perfusion territories of the right ICA (blue), left ICA (red) and the basilar artery (green). The caudate nucleus on the right side is supplied by the contralateral ICA (besides white star). The lentiform nuclei on both sides are supplied by their respective ipsilateral ICA. Furthermore, both thalami are partially supplied by their ipsilateral ICA and basilar artery. Circle of Willis on 2D phase-contrast MR angiogram (MRA), phase encoded in the right-left direction $\mathbf{b}$ and anterior-posterior direction c. Blood flowing to the patient's right and anterior direction is black and blood flowing to the patient's left and posterior direction is white. There is collateral flow towards the right hemisphere via the right A1 segment (retrograde flow, black in $\mathrm{B}$, white in $\mathrm{C}$, indicated by white star). Maximum intensity projection $\mathbf{d}$ shows the anatomy of the circle of Willis 
Table 2 Prevalence of various types of blood supply to the deep brain structures
$I C A$ internal carotid artery; $B A$ basilar artery; the deep brain structures were supplied by more than one artery in some patients. ${ }^{a}$ indicates a significant difference compared to healthy control subjects. $(p<0.03$, two-sided Fishers' exact test)

\begin{tabular}{lccc}
\hline & Ipsilateral ICA & Contralateral ICA & BA \\
\hline Caudate nucleus & & & - \\
Healthy controls & $18(100 \%)$ & $0(0 \%)$ & - \\
Symptomatic patients & $15(100 \%)$ & $4(27 \%)^{\mathrm{a}}$ & - \\
Asymptomatic patients & $19(95 \%)$ & $1(5.0 \%)$ & $1(5.6 \%)$ \\
Lentiform nucleus & $18(100 \%)$ & - & $1(6.7 \%)$ \\
Healthy controls & $15(100 \%)$ & - & $0(0 \%)$ \\
Symptomatic patients & $20(100 \%)$ & - & $16(89 \%)$ \\
Asymptomatic patients & $15(83 \%)$ & $2(13 \%)$ & $14(93 \%)$ \\
Thalamus & $12(80 \%)$ & $2(10 \%)$ & $16(80 \%)$ \\
Healthy controls & $15(75 \%)$ & & \\
Symptomatic patients & & & \\
Asymptomatic patients & & & \\
\end{tabular}

In one of the five patients in whom the caudate nucleus was supplied by the contralateral ICA, anterior collateral flow was not detected. This may be explained by supply of part of the caudate nucleus by the recurrent artery of Heubner. This artery originates from the anterior cerebral artery close to its junction with the anterior communicating artery and although hard to detect on MR angiography, is present in almost all hemispheres examined in post-mortem studies [22, 23]. Blood flow from the contralateral ICA via the recurrent artery of Heubner to the caudate nucleus is therefore not necessarily accompanied by detectable anterior collateral flow via the circle of Willis. This illustrates the importance that perfusion territory selective ASL-MRI might in comparison with MRA.

In both the healthy control subjects and patients with a symptomatic ICA stenosis our results show that the lentiform nucleus can be supplied by either the ipsilateral ICA or the posterior circulation. A previous study that investi- gated the effect of anatomical features of the circle of Willis on the perfusion territory patterns in the deep brain structures found that the lentiform nucleus was supplied by the contralateral ICA in $6 \%$ of patients with cerebral ischemia without carotid artery stenosis [24]. In none of the patients in our study the lentiform nucleus received blood from the contralateral ICA. This apparent discrepancy can be explained by chance or by the fact that in the patients from the previous study, occlusions in the anterior and middle cerebral artery may have resulted in recruitment of (leptomeningeal) collateral blood flow to the lentiform nucleus via the contralateral carotid artery. In addition to the different patient populations, the perfusion territory selective ASL labeling techniques also differed.

No differences were found between the patients with ICA stenosis and the healthy control subjects with respect to the supply routes to the thalamus. We did find a large inter-subject variation of the perfusion contributions to the

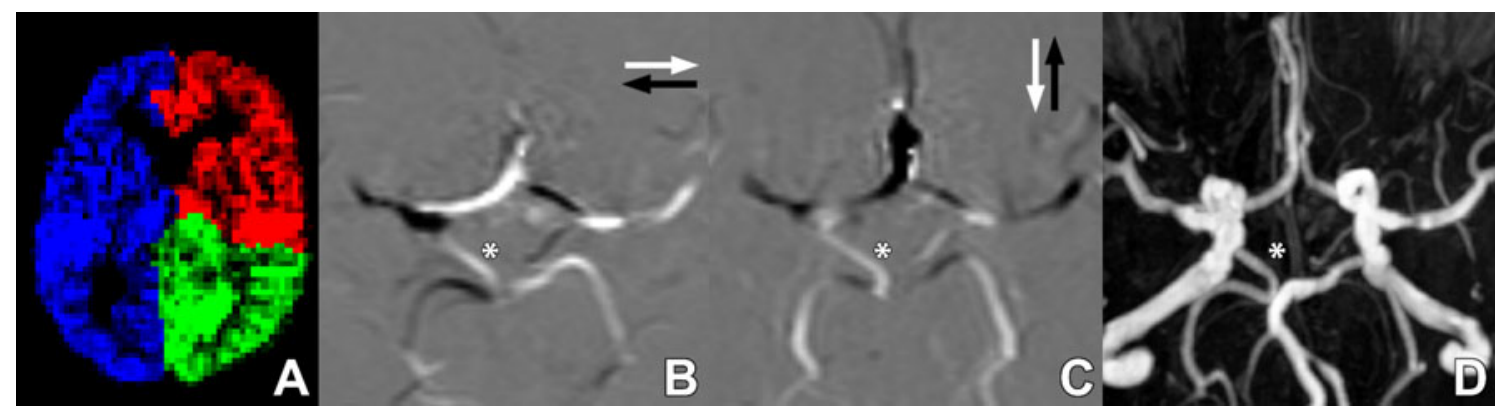

Fig. 3 Perfusion territory image a of a patient with a right-sided symptomatic ICA stenosis and a right-sided unilateral fetal-type variant of the circle of Willis. Colors represent the perfusion territory of the right ICA (blue), left ICA (red) and the basilar arteries (green). The caudate nuclei and lentiform nuclei on both sides are supplied by their respective ipsilateral ICA. Furthermore, the thalamus on the left side is partially supplied by both its ipsilateral ICA and basilar artery, while the thalamus on the right side (symptomatic side) is completely supplied by its ipsilateral ICA. Circle of Willis on 2D phase-contrast
MRA, phase encoded in the right-left direction $\mathbf{b}$ and anteriorposterior direction c. Blood flowing to the patient's right and anterior direction is black and blood flowing to the patient's left and posterior direction is white. Phase contrast MRAs show anterior to posterior flow through the right posterior communicating artery (besides white star; white in $\mathbf{b}$ and $\mathbf{c})$. Maximum intensity projection $\mathbf{d}$ shows a rightsided fetal-type posterior communicating artery (besides white star), which explains why the right-sided thalamus is not supplied by the basilar artery 
thalamus in both the patients and healthy control subjects. The thalamus is predominantly supplied via the basilar artery, but may also receive blood from the ipsilateral ICA. In a few subjects, the contralateral ICA also contributed to the perfusion of the thalamus. Textbook-based perfusion territory maps do not give this unique perfusion territory information in individual subjects. Because the supply routes of the thalamus varied the most of all the nuclei in our study, perfusion territory selective ASL-MRI may be particularly useful for the thalamus.

A limitation of this study is that in patients with an ICA stenosis, there may be a delayed arrival of the bolus of magnetically labeled blood. As the perfusion-weighted images in ASL are acquired after a fixed amount of time, this may potentially lead to reduced sensitivity for delayed perfusion via collaterals. In order to compensate for underestimation of collateral blood flow we employed a pseudo-continuous ASL labeling technique, where the protons labeled directly after the start of the labeling have an effective delay time of more than $3 \mathrm{~s}$. As the collateral blood flow paths via the circle of Willis are relatively fast and have shown to not result in a measurable delayed arrival of the magnetically labeled blood bolus in a previous ASL-MRI study using multiple time intervals [25], this should be adequate. Another limitation of this study is the relatively small group sizes. With a larger study population smaller differences in prevalence of perfusion contributions to the various nuclei may have been detected between the patient groups and healthy control subjects. Still, with the size of the current study we could demonstrate a difference in perfusion contributions to the caudate nucleus between patients with a symptomatic carotid artery stenosis and healthy control subjects.

Our results show that in patients with a symptomatic ICA stenosis, the pattern of perfusion territories may be altered with a higher prevalence of perfusion contribution to the caudate nucleus from the contralateral ICA. Perfusion territory selective ASL-MRI may facilitate treatment decisions in patients with steno-occlusive carotid artery disease by providing information on the perfusion territories and by identifying the symptomatic artery.

Open Access This article is distributed under the terms of the Creative Commons Attribution Noncommercial License which permits any noncommercial use, distribution, and reproduction in any medium, provided the original author(s) and source are credited.

\section{References}

1. Hendrikse J, Petersen ET, Cheze A, Chng SM, Venketasubramanian N, Golay X (2009) Relation between cerebral perfusion territories and location of cerebral infarcts. Stroke 40:1617-1622
2. van der Zwan A, Hillen B, Tulleken CAF, Dujovny M, Dragovic L (1992) Variability of the territories of the major cerebral arteries. J Neurosurg 77:927-940

3. van Laar PJ, Hendrikse J, Golay X, Lu H, van Osch MJ, van der Grond J (2006) In vivo flow territory mapping of major brain feeding arteries. Neuroimage 29:136-144

4. Aitken HF (1928) Diagram of arterial circulation of the basal ganglia. New Engl J Med 199:2084

5. Liebeskind DS (2003) Collateral circulation. Stroke 34:2279-2284

6. Tatu L, Moulin T, Bogousslavsky J, Duvernoy H (1998) Arterial territories of the human brain: cerebral hemispheres. Neurology 50:1699-1708

7. Lee JS, Lee DS, Kim YK, Kim J, Lee HY, Lee SK, Chung JK, Lee MC (2004) Probabilistic map of blood flow distribution in the brain from the internal carotid artery. Neuroimage 23:14221431

8. Jones CE, Wolf RL, Detre JA, Das B, Saha PK, Wang J, Zhang Y, Song HK, Wright AC, Mohler EM 3rd, Fairman RM, Zager EL, Velazquez OC, Golden MA, Carpenter JP, Wehrli FW (2006) Structural MRI of carotid artery atherosclerotic lesion burden and characterization of hemispheric cerebral blood flow before and after carotid endarterectomy. NMR Biomed 19:198-208

9. van Laar PJ, Hendrikse J, Klijn CJ, Kappelle LJ, van Osch MJ, van der Grond J (2007) Symptomatic carotid artery occlusion: flow territories of major brain-feeding arteries. Radiology 242:526-534

10. Paiva FF, Tannus A, Silva AC (2007) Measurement of cerebral perfusion territories using arterial spin labelling. NMR Biomed 20:633-642

11. van Laar PJ, van der Grond J, Hendrikse J (2008) Brain perfusion territory imaging: methods and clinical applications of selective arterial spin-labeling MR imaging. Radiology 246:354-364

12. Simons PC, Algra A, van de Laak MF, Grobbee DE, van der Graaf Y (1999) Second manifestations of ARTerial disease (SMART) study: rationale and design. Eur J Epidemiol 15:773-781

13. Nederkoorn PJ, Mali WP, Eikelboom BC, Elgersma OE, Buskens E, Hunink MG, Kappelle LJ, Buijs PC, Wüst AF, van der Lugt A, van der Graaf Y (2002) Preoperative diagnosis of carotid artery stenosis: accuracy of noninvasive testing. Stroke 33:2003-2008

14. Gevers S, Nederveen AJ, Bokkers RP, Hendrikse J, Kies DA, Teeuwisse WM, Majoie CB, van Osch MJ (2009) Reproducibility of flow territories defined by plannings-free vessel encoded pseudo-continuous arterial spin labeling. ISMRM 17th Scientific Meeting Proceedings 1524.

15. Wong EC (2007) Vessel-encoded arterial spin-labeling using pseudocontinuous tagging. Magn Reson Med 58:1086-1091

16. Dai W, Garcia D, de Bazelaire C, Alsop DC (2008) Continuous flow-driven inversion for arterial spin labeling using pulsed radio frequency and gradient fields. Magn Reson Med 60:1488-1497

17. Wu WC, Fernandez-Seara M, Detre JA, Wehrli FW, Wang J (2007) A theoretical and experimental investigation of the tagging efficiency of pseudocontinuous arterial spin labeling. Magn Reson Med 58:1020-1027

18. Ye FQ, Frank JA, Weinberger DR, McLaughlin AC (2000) Noise reduction in $3 \mathrm{D}$ perfusion imaging by attenuating the static signal in arterial spin tagging (ASSIST). Magn Reson Med 44:92-100

19. Rutgers DR, Klijn CJ, Kappelle LJ, van Huffelen AC, van der Grond $\mathrm{J}$ (2000) A longitudinal study of collateral flow patterns in the circle of Willis and the ophthalmic artery in patients with a symptomatic internal carotid artery occlusion. Stroke 31:1913-1920

20. Wong EC, Kansagra AP (2008) Mapping Middle Cerebral Artery Branch Territories with Vessel Encoded Pseudo-Continuous ASL: Sine/Cosine Tag Modulation and Data Clustering in Tagging Efficiency Space. ISMRM 16th Scientific Meeting Proceedings 182. 
21. Chng SM, Petersen ET, Zimine I, Sitoh YY, Lim CC, Golay X (2008) Territorial arterial spin labeling in the assessment of collateral circulation: comparison with digital subtraction angiography. Stroke 39:3248-3254

22. Boongird A, Duangtongphon P (2009) Variation of the recurrent artery of Heubner in human cadavers. J Med Assoc Thai 92:643-647

23. Loukas M, Louis RG Jr, Childs RS (2006) Anatomical examination of the recurrent artery of Heubner. Clin Anat 19:25-31
24. Hendrikse J, Petersen ET, Chng SM, Venketasubramanian N, Golay X (2010) Distribution of cerebral blood flow in the nucleus caudatus, nucleus lentiformis, and thalamus: a study of territorial arterial spin-labeling MR imaging. Radiology 254:867875

25. Bokkers RP, van der Worp HB, Mali WP, Hendrikse J (2009) Noninvasive MR imaging of cerebral perfusion in patients with a carotid artery stenosis. Neurology 73:869-875 